\title{
Segregación Educativa en Tiempos de Pandemia: Balance de las Acciones Iniciales durante el Aislamiento Social por el Covid-19 en Argentina
}

\author{
Educational Segregation in Times of Pandemic: Balance of \\ Initial Actions during Social Isolation by Covid-19 in Argentina
}

\author{
Marisa Álvarez * \\ Natalia Gardyn \\ Alberto Iardelevsky \\ Gabriel Rebello
}

Universidad de Buenos Aires, Argentina

\begin{abstract}
La Argentina ha implementado medidas de aislamiento social obligatorio inicialmente previsto para un período de quince días y luego prorrogado, recién iniciado el año escolar. Este artículo brinda una aproximación a las estrategias de gestión que se desarrollaron ante el contexto de aislamiento obligatorio, un análisis de los primeros instrumentos normativos, su relación con el desarrollo curricular, y sus efectos en términos de segregación educativa. Se trata de un estudio exploratorio y descriptivo, utilizando un método cualitativo, a partir de análisis documental y entrevistas a protagonistas del sistema educativo que debieron implementar las políticas en dicho contexto. La preexistencia de brechas en el acceso a recursos digitales, la invisibilización de las condiciones reales de la población, las decisiones vertiginosas de los niveles de gobierno centrales combinado con la heterogeneidad de las resoluciones a nivel institucional y a nivel de grupo escolar evidencian aspectos de profundización de la segmentación educativa.
\end{abstract}

Descriptores: Derecho a la educación; Segregación escolar; Administración de la educación; Plan de estudio; Aprendizaje en línea.

\begin{abstract}
Within a few days after the beginning of the school year, Argentina has implemented mandatory isolation measures that were initially announced for a period of fifteen days and then extended. This paper approaches the management strategies developed in under mandatory isolation, an analysis of the initial regulations related to curricular development, and its effects in terms of educational segregation. This paper consists in an exploratory and descriptive study which uses qualitative methods, based on documentary analysis and interviews with actors in the educational system who had to carry out policies in such circumstances. Preexistence of gaps in the access to digital resources, invisibility of real living conditions, dizzying decisions at central government levels combined with the heterogeneity of solutions at the institutional level and at the school group level shows aspects of a deepening in the educational segregation.
\end{abstract}

Keywords: Rights to education; School segregation; Educational administration; School curriculum; Online learning.

*Contacto: malvarez@untref.edu.ar

ISSN: 2254-3139

www.rinace.net/riejs/

revistas.uam.es/riejs
Recibido: $\quad 2$ de abril 2020

$1^{\text {a }}$ Evaluación: 17 de abril 2020

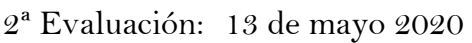

Aceptado: 25 de mayo 2020 


\section{Introducción}

América Latina es la región más desigual del planeta (Kliksberg, 2005), situación que se manifiesta en todas las facetas de la vida cotidiana. La Argentina tuvo una experiencia de progreso económico y social a mediados del siglo pasado, pero hoy en día no sólo no ha crecido económicamente, sino que además muestra signos de una sociedad fragmentada y con una fuerte desigualdad (Bonfiglio, Veram y Salvia, 2019; Kessler, 2014), que se evidencia en un coeficiente de Gini cercano al 0,5.

Esta desigualdad se posicionó como un problema central de las políticas públicas. En los últimos años, resultado de un relevante movimiento de ampliación de derechos educativos, se logró un importante aumento de la cobertura, en particular, en el nivel medio (al 2014, la tasa neta de escolarización de la educación secundaria alcanzaba el 86,63, siendo de 81,44 en $\left.2000^{1}\right)$. No obstante, los resultados educativos -medidos con distintos instrumentos y metodologías- muestran la gran disparidad en los indicadores, en particular cuando se analiza por nivel socioeconómico de la población. Por ejemplo, en el último operativo $\mathrm{PISA}^{2}$, que mostró un rendimiento mediocre, lo más preocupante fue la alta dispersión en los resultados. Asimismo, las estadísticas educativas del Ministerio de Educación de la Nación dan cuenta de la gran diferenciación en el rendimiento de acuerdo al estrato social de pertenencia.

En este marco, un evento sanitario, mundial e inesperado, ha desencadenado, la interrupción forzosa del dictado de clases presenciales y un conjunto de acciones dispuestas por las autoridades nacionales, provinciales e institucionales para el mantenimiento de cierto orden de continuidad, en el que se recurrió en primer término al uso de tecnologías para sustituir la presencialidad. Este documento hace un análisis de las primeras intervenciones y los efectos que estas acciones pudieran generar sobre la segmentación educativa ya existente.

\section{Antecedentes conceptuales}

En Argentina existe una copiosa investigación en relación con el tema de fragmentación y segmentación educativa. Los primeros trabajos sobre segmentación social del sistema educativo se remontan a 1983 (Braslavsky, 1985) y luego a los trabajos de Kessler (2002, 2014), Tiramonti (2004), Gasparini y otros (2011), Krüger (2013, 2019), etc. En general, estos trabajos abordan el tema de la segmentación educativa desde una perspectiva sociológica, entendida como "la distribución desigual de los estudiantes en los centros educativos según sus características personales o sociales" (Murillo, 2016, p. 35), dando cuenta de los circuitos diferenciales de acceso y calidad educativa según nivel socioeconómico. Estos estudios, en general, muestran -con suficiente evidencia empíricala importante distancia de acceso a la educación de calidad según sector social de pertenencia. No sólo dan cuenta de este acceso diferencial, sino también de los circuitos y recorridos diferenciales que realizan los estudiantes en sus trayectorias educativas.

\footnotetext{
${ }^{1}$ Siteal, en https://www.siteal.iiep.unesco.org/indicadores, en base a la Encuesta Permanente de Hogares del INDECArgentina.

${ }^{2}$ Informe del Programa Internacional para la Evaluación de Estudiantes (PISA, por sus siglas en inglés: Programme for International Student Assessment), llevado a cabo por la OCDE a nivel mundial que mide el rendimiento académico de los estudiantes de 15 años en matemáticas, ciencia y lectura.
} 
Murillo y Martínez-Garrido (2017) abordan dos tipos de segregación escolar: la segregación por nivel socio-económico y la segregación cultural. En el contexto de aislamiento social, ambos se evidencian tanto en las condiciones de partida de las escuelas (de los docentes y de los alumnos) para desarrollar una estrategia alternativa a la presencialidad, como en las posibilidades simbólicas de incluirse en las propuestas escolares relacionadas con el capital cultural familiar.

Esta segregación escolar por nivel socio-económico (donde los estratos sociales se concentran en instituciones distintas) y por la distribución de bienes materiales y culturales que se ven atravesados por la misma situación de injusticia y desigualdad, remiten a lo que la literatura especializada enuncia como desigualdad de acceso, dado que los alumnos no están recibiendo propuestas educativas similares ni en calidad ni en volumen. Se trata sólo de igualdad legal y formal (Marchesi, 2000). Como expresan Valenzuela, Bellei y Ríos (2014), en general, la evidencia respalda que la segregación escolar socioeconómica es una característica relevante de los sistemas educativos y que, en consecuencia, las políticas del sector pueden discutir en términos de su contribución potencial para su aumento o disminución.

La segregación escolar por nivel socioeconómico se manifiesta en la pérdida de la escuela como espacio de intercambio de capital social y formación de redes heterogéneas. En este sentido, se amplía la brecha de desigualdad. Así, puede afirmarse que la segregación escolar también manifiesta la injusticia social por las condiciones desfavorables de inclusión y no tanto por la exclusión. (Sen y Kliksberg, 2007). El acceso a la escuela en condiciones desfavorables impide participar integralmente de experiencias de escolaridad marcadas por el distanciamiento social. Desde esta perspectiva y dentro del contexto de análisis puede afirmarse que las herramientas digitales que se promueven se basan en una lógica individual y técnica. Esta situación crea un obstáculo que impide sustituir las experiencias colectivas que la escuela tradicional promueve. Como afirma Meirieu (2020) "cada uno frente a su propia pantalla y en mutua indiferencia, consumen software en lugar de compartir conocimientos". Por lo cual, frente a la situación de aislamiento social, sumado a la falta de acceso a la tecnología, se profundiza la desigualdad entre sectores y aumentan las barreras para la integración educativa.

El contexto de aislamiento social exige definiciones de política curricular. El currículum puede trascender, siguiendo a Angulo (1994), al currículum oficial, de modo ser analizado como una realidad interactiva, es decir la forma concreta en que se realiza en las aulas. En este sentido, amerita la explicitación de un currículum básico esencial que otorgue nitidez a aquellos contenidos irrenunciables constituyéndose en un referente para la gestión curricular escolar. La situación impone interrogantes en el campo curricular vinculados a la preocupación por la selección cultural que es propia del establecimiento de un currículum común: ¿Esta selección satisface las necesidades, valoraciones y utilidades de los diferentes grupos sociales? ¿Lo común invisibiliza la diferencia cuando la experiencia escolar en el aislamiento se presenta como una colección de ejercitaciones para su resolución? ¿Cómo se resuelve la tensión entre lo común y lo diverso en un contexto de emergencia cuando en el Estado se concentran los esfuerzos para garantizar el acceso a propuestas educativas que impiden la interrupción de una cotidianeidad en la que el aprendizaje cuente con un espacio y tiempo determinado?

Pero como hemos mencionado, no es el único nivel de realización del curriculum. Es cierto que, en esta situación, éste se caracterizaría por sus limitaciones, tanto en relación con el 
control de la enseñanza a cargo de los docentes, como por la restricción de las interacciones entre profesores y alumnos y alumnos entre sí. Esto se profundiza, dado que una de las consecuencias que impone el aislamiento es la reducción de los márgenes de decisiones institucionales y de docentes. ¿Cuáles son las posibilidades de las escuelas y los docentes de programar su enseñanza cuando estos no disponen ni de los dispositivos ni de los materiales habituales del aula? Como fue señalado en las respuestas relevadas. Es aquí donde las diferencias sociales, económicas y culturales se tornan en puentes o barreras en relación con la accesibilidad y el derecho a la educación. Este es el foco de atención donde debiera asentarse el debate teórico acerca del campo curricular y también el de la política y administración del currículum, en una situación de la gravedad como la actual.

En este sentido, es importante comprender la importancia del contenido curricular, no como enunciación temática, sino como práctica cultural de significación. El curriculum encuentra su sentido en las actividades de enseñanza, fundamentalmente en las interacciones entre docentes y estudiantes preocupados por los aprendizajes. Esto se expresa en la organización de las normas que los regulan, en los tipos de agrupamientos y en las intervenciones docentes. En la actividad presencial, el espacio de participación se encuentra atravesado por la inmediatez de la acción que es constitutiva de la naturaleza del aula. Ahora bien, en las propuestas a distancia existe una pérdida o transmutación de los espacios de interacción que deben ser considerados por quienes administran las propuestas de enseñanza. Esa pérdida se torna más profunda cuando los estudiantes destinatarios no cuentan con los recursos suficientes para desplegar todas sus potencialidades, inquietudes, dudas y formas de resolución adoptadas. La segregación en su forma cultural aumenta en su dimensión.

\subsection{Educación y emergencia sanitaria}

La emergencia sanitaria requirió de estrategias para hacer frente a una situación inédita. En el sector educación, la interrupción de las formas de cotidianeidad escolar se constituyó en una medida inevitable y las administraciones educativas debieron recurrir a distintas estrategias para dar continuidad al período lectivo. Las opciones desplegadas por las distintas instancias del gobierno para paliar el aislamiento, y asegurar la comunicación entre los diversos actores institucionales, estuvieron basadas en las tecnologías informáticas y de comunicación.

Previo a la pandemia, algunos autores ya anticipaban nuevos escenarios para los sistemas educativos, en el cual la tecnología se constituiría en la llave maestra para resolver el tema de la educación, incluso en su versión más extrema con prescindencia de los docentes y de las estructuras organizacionales escolares tal como las conocemos. Es decir, aventuraron un escenario sin su soporte material espacial. Definían a la escuela como una tecnología vieja que debiera ser reemplazada por otra nueva (Cobo, 2016; Piscitelli, 2009). Esta línea de pensamiento aboga por un sistema de enseñanza por computadoras, ya que con desarrollos de inteligencia artificial las computadoras podrían evaluar el rendimiento escolar, aún en asignaturas complejas. Sin embargo, como señalan Sunkel, Trucco y Espejo (2014), los obstáculos para el uso de las TIC en las escuelas no se refieren solamente al aspecto de la tecnología, sino que comprenden además del acceso, el uso de los recursos para la enseñanza y la incorporación a la gestión de las escuelas.

Por lo tanto, no puede obviarse la referencia a la presencia de la tecnología de la información en la escuela como el resultado de las nuevas políticas educativas. Esta incorporación es productora de tensión entre lo que se denomina escuela digital -como un 
nuevo paradigma que se propone a partir del desarrollo tecnológico (un encantamiento digital)- y la escuela digitalizada como la respuesta que pueden articular algunas escuelas en la que se hacen presente la burocratización, la normativación, el uso tradicional de los nuevos medios y una tecnificación por encima de la pedagogía. Especialistas de Argentina como Mariana Maggio (2012, 2019), Teresa Lugo (2016) y Andrea Brito (2015), entre otros, han propuesto un análisis más complejo, en términos socioeducativos, respecto de la utilización de las tecnologías en educación al considerar las ventajas que puede traer su aprovechamiento para mejorar sustantivamente la educación. Sin embargo, advierten sobre el reconocimiento del contexto de aplicación de las tecnologías y fundamentalmente, de los sujetos.

Un antecedente importante en nuestro país fue el Programa Conectar Igualdad, lanzado en $2010^{3}$ bajo el modelo 1 a 1 (una computadora por alumno), que consistió en la entrega de netbooks a alumnos y docentes de escuelas de gestión estatal del nivel secundario y a alumnos de la modalidad de educación especial y de institutos de formación docente entre el 2011 y el 2014 (Marés Serra, 2012).

\subsection{Planteo del problema}

La emergencia de una crisis global como la actual plantea las preguntas: ¿Están las escuelas adecuadamente preparadas para enfrentar esta crisis? ¿Existen adecuados mecanismos para continuar con la tarea pedagógica de manera no presencial siendo que parten de condiciones desiguales? ¿Qué atención especial requieren los contextos vulnerables? ¿Cómo abordan los docentes estos nuevos requerimientos?, ¿Qué efectos tiene sobre la segregación escolar?

El objetivo de este trabajo es hacer una primera aproximación, que comprende los días previos al aislamiento y la primera etapa de quince días, prestando particular atención a las estrategias de gestión que desarrollaron tanto autoridades como establecimientos frente al contexto de cuarentena obligatoria, y sus posibles efectos en términos de segregación educativa.

\section{Metodología}

El estudio que se presenta es de carácter exploratorio y descriptivo, que pretende una primera sistematización de las acciones desarrolladas por autoridades e instituciones, a partir de fuentes primarias y secundarias. Se puede definir en términos generales al caso, siguiendo la formulación de Neiman y Quaranta (2006), como el recorte empírico de un hecho, proceso, grupo o institución social de una realidad social más amplia, en función de la comprensión de un tema o problema de investigación.

En este trabajo se realizó un recorte temporal (las acciones llevadas a cabo desde los niveles centrales y desde el grupo de escuelas seleccionadas en los días inmediatamente previos al decreto de aislamiento obligatorio y en los primeros quince días) y espacial

\footnotetext{
${ }^{3}$ De acuerdo al Informe presentado por el Ministerio de Educación "Panorama regional de estrategias uno a uno: América Latina + el caso de Argentina”, se proyectó entregar en el marco del Programa Conectar Igualdad 3.600.000 netbook con el objetivo de "llegar a una sociedad alfabetizada en las nuevas tecnologías de la información y la comunicación (TIC), con la posibilidad de un acceso democrático a recursos tecnológicos e información sin distinción de grupo social o económico, ni de densidad poblacional, alcanzando las más diversas geografías, tanto rurales como urbanas”. De acuerdo a este informe, algunos gobiernos provinciales (como la Ciudad Autónoma de Buenos Aires, San Luis, Buenos Aires) también implementaron políticas de provisión de equipos en el nivel primario.
} 
(escuelas pertenecientes al área metropolitana de Buenos Aires), seleccionando dentro de dicho universo instituciones del sector público y privado y que atiendan a grupos de clase baja, media y alta. Los criterios con que fueron seleccionadas las escuelas, fue por localización y características de población que atienden, así como también por el tipo de gestión, estatal o privada. La selección de escuelas fue intencional.

Se seleccionaron tres escuelas públicas y cuatro privadas de diferentes características. De las tres primeras, dos están localizadas en villas de emergencia y su población es de clase baja; la tercera atiende a alumnos de familias de clase media y se encuentra en un barrio de la Ciudad Autónoma de Buenos Aires (CABA). Las cuatro escuelas privadas son: una perteneciente a una comunidad religiosa localizada en un barrio del centro geográfico de la CABA. Atiende una población de clase media y media alta. Otra cuya población es de clase alta, ubicada en un barrio acomodado de la CABA, las otras dos instituciones, localizadas en el primer cordón del conurbano de la provincia de Buenos Aires, atienden población de clase media y media baja. Los criterios de selección de las instituciones incluyeron: la ubicación, tipo de gestión y población que atiende.

El estudio comprendió en primer término el análisis de documentación con el objetivo de analizar el devenir de las acciones de política y su correspondiente concretización. Al respecto se consideraron los Decretos presidenciales, las Resoluciones del Ministerio Nacional, y Resoluciones del Ministerio de Educación y Comunicados de autoridades nacionales y de la CABA referidas al tema, entre el 3 y el 31 de marzo de 2020.

El trabajo de campo se completó con entrevistas a docentes y directivos, en tanto protagonistas de la implementación de las políticas educativas de las jurisdicciones. A tales efectos, se construyó un cuestionario que les fue aplicado para abordar los siguientes temas: características de las escuelas, acciones tomadas, disponibilidad de tecnología y su mediación y rol de directivos y de la administración del sistema educativo.

\section{Resultados}

\subsection{Una breve cronología}

El 3 de marzo de 2020 el Ministerio de Salud de la Nación reportó el primer caso positivo de coronavirus en Argentina, ese mismo día, de acuerdo al calendario escolar, se daba inicio el Ciclo Lectivo 2020 en prácticamente todas las escuelas del país. Tres días después el Ministerio de Educación de la Nación emitió la Resolución Nro. 82-MEN/2020, con un protocolo para suspensión de clases en aulas o escuela que presenten enfermos confirmados, basado en la hipótesis de viajeros llegados de países con presencia del virus.

A tan solo dos semanas del inicio, el 15 de marzo, el presidente de la Nación anunció la suspensión de clases en todo el país ${ }^{4}$. El Ministerio de Educación estableció que,

durante el plazo que dure la suspensión de asistencia de estudiantes, el personal docente, no docente y directivo concurrirá normalmente a los efectos de mantener el desarrollo habitual de las actividades administrativas, la coordinación de los servicios

\footnotetext{
${ }^{4}$ Res. 108/2020. Durante esas dos semanas, cada escuela fue aplicando las medidas sugeridas por el Ministerio de Salud a través de comunicados del Ministerio de Educación. Mediante Resolución Ministerial N 82/2020 se recomendaron las medidas preventivas a aplicar al 6 de marzo pasado, y asimismo por Resolución 103/2020 se estableció un protocolo a aplicar ante la aparición de diferentes situaciones en el ámbito educativo en general.
} 
sociales y las actividades pedagógicas que se programen para el presente período de excepcionalidad.

Como así también,

\begin{abstract}
asegurar las medidas necesarias para la comunicación y el seguimiento de las actividades de enseñanza propuestas por las autoridades educativas nacionales y jurisdiccionales, que estarán disponibles para su implementación durante este período mediante distintos soportes, a los efectos de acompañar la vinculación entre los equipos docentes, estudiantes, familias y comunidades.
\end{abstract}

En forma concurrente se creó, mediante Resolución del Ministerio de Educación $\mathrm{N}^{\circ}$ 106/2020, el Programa "Seguimos Educando", fundamentado en que "el rol de la escuela resulta irremplazable, pero entendiendo que, en este excepcional contexto, resulta necesario llevar adelante para hacer efectivo el derecho a la educación, un programa que facilite el acceso a contenidos educativos y bienes culturales hasta tanto se supere la emergencia”.

Los objetivos del Programa Seguimos Educando son: a) colaborar con las condiciones para la continuidad de las actividades de enseñanza; b) asegurar la distribución de los recursos y/o materiales; c) Elaborar materiales y recursos según los Núcleos de Aprendizaje Prioritarios; y d) Elaborar y difundir materiales y recursos culturales para el uso familiar y comunitario.

Entre las acciones definidas en el marco del Programa, se realizó la puesta en línea de una plataforma (seguimoseducando.gob.ar) que incluye recursos de autoaprendizaje, sugerencias para familias y docentes, películas, entrevistas, propuestas didácticas y de comunicación a través de redes sociales y herramientas de videoconferencia, propuestas para el tiempo libre y agenda de eventos en línea.

El Programa también previó la producción y emisión de la programación audiovisual a través de seis señales dependientes de la Secretaría de Medios y Comunicación Pública. ${ }^{5}$ En el Portal www.educ.ar se pusieron a disposición recursos educativos, secuencias didácticas y propuestas formativas. El Instituto Nacional de Formación Docente, dependiente del Ministerio de Educación, organizó cursos para que los docentes aprendan a usar herramientas digitales. Para las comunidades educativas sin acceso a internet (en situación de aislamiento, ruralidad y contextos de alta vulnerabilidad social) se previó la producción y distribución de material impreso.

El lunes 16 de marzo, primer día de suspensión de clases, los docentes tuvieron que asistir a las escuelas, donde se realizaron reuniones con los directivos para elaborar estrategias en función del contexto comunitario. En las escuelas públicas, se implementaron turnos de guardia para atender los comedores escolares.

Frente a las dificultades para sostener la continuidad de las actividades de enseñanza, y en particular, la comunicación con los estudiantes, el Ministerio de Educación fue elaborando acciones complementarias a las planificadas para fortalecer la vinculación: recomendaciones para docentes, convocatoria a los Centros de Estudiantes para integrar la comisión consultiva ${ }^{6}$ del Programa Seguimos Educando, incorporación de la radio como

\footnotetext{
${ }_{5}^{5}$ Las seis señales son: Televisión Pública Argentina y sus repetidoras, Encuentro, Paka Paka, DeporTV, Radio Nacional y Cont.ar. Está estructurada en dos bandas horarias de dos horas, destinadas una al nivel primario y otro al medio.

${ }^{6}$ El artículo $5^{\circ}$ de la Resolución del Ministerio de Educación que crea el Programa Seguimos Educando establece que la Comisión Consultiva del Programa está conformada por: Ministro de Educación (quien la preside) a. DOS (2)
} 
estrategia para hacer llegar contenidos educativos a los hogares que presentan limitaciones de acceso a puntos de conectividad.

En el caso del Gobierno de la CABA, el Ministerio de Educación, recién el 26 de marzo elaboró un documento de Lineamientos para Supervisiones Escolares y Equipos de Conducción durante el período de suspensión de clases presenciales. Los lineamientos se centraron en: 1) Gestión escolar en un esquema de guardia, 2) Comunicación con las familias, 3) Continuidad pedagógica. Asimismo, se ofrecieron recursos complementarios a aquellos que las escuelas ya estaban utilizando: a través de la Plataforma Mi Escuela, organización de Aulas Virtuales que permitan gestionar actividades relacionadas con la formación académica no presencial (que a esa fecha no estaban disponibles) y la Plataforma de Inglés adaptativa: a través del portal.bue.edu.ar.

El gobierno nacional mantuvo una estrategia comunicacional centrada en los máximos niveles, con comunicados del Presidente a la población (algunos en cadena nacional) y del Ministro de Educación. Ese mismo 26 de marzo, el presidente respondió en una entrevista televisiva que no era urgente el reinicio de clases, medida que guarda relación con las recomendaciones médicas de retomar paulatinamente las actividades post cuarentena. Y el 30 de marzo en reunión virtual con la comisión de educación de la Cámara de Diputados, el ministro de Educación manifestó que no había fecha para el reinicio de las clases, así como que esta vuelta podría darse de forma escalonada según las condiciones de cada provincia, así como que es posible la articulación del ciclo lectivo de este año con el del 2021.

\subsection{La urgencia del devenir y el planeamiento de las acciones}

Frente a este estado de situación, con las primeras decisiones tomadas y las acciones puestas en marcha, se generaron debates y discusiones respecto de la mejor forma de dar continuidad al ciclo educativo, considerando el nivel de desarrollo de los sistemas de enseñanza por computadoras en el país. La situación de emergencia es claramente una excepcionalidad. En temas de planificación, nos podemos preguntar acerca de cuán preparados estamos para situaciones de crisis. Ante la emergencia, se priorizan ciertas decisiones críticas, que no pueden demorarse, incluyendo la implementación de acciones y uso de recursos para darles respuestas.

Nuestros entrevistados dieron cuenta de esta urgencia y el desvío de las acciones de desarrollo educativo, frente a las necesidades materiales concretas, como es la entrega de viandas alimentarias. Las primeras resoluciones establecían suspensión de clases con apertura de los comedores. La prioridad fue asegurar las necesidades básicas.

El tema de las viandas es otra movida...garantizar alimento a las familias...Las viandas, un desastre. Los camiones llegan tarde, el primer día hubo la mitad de las raciones, entonces las familias fueron y se quedaron sin comida... todo muy dificil. (E1: Maestra 1er.grado, escuela pública, localización villa de emergencia, clase social baja, CABA)

Esta es una primera situación de profundización de la segmentación educativa: Aquellas instituciones que atienden población vulnerable, la prioridad está puesta en asegurar la

funcionarios/as del ministerio de educacion con jerarquía no inferior a Subsecretaria/o; b. La Secretaria General y CINCO (5) representantes del consejo federal de educación, uno por cada región; c. un/a (1) representante de educ.ar sociedad del estado. d. UN/A (1) representante por cada una de las siguientes entidades sindicales: CTERA, UDA, CEA, SADOP y AMET. 
alimentación de los estudiantes. La organización institucional escolar estuvo puesta en la necesidad de resolver cuestiones de gestión administrativa sobre los alimentos antes que el plan de desarrollo curricular. Se evidencia los efectos de la segregación por nivel socioeconómico sobre la segregación cultural. Es decir, la situación descrita da cuenta de una doble segregación.

Uno de los aspectos que surgieron más relevantes ha sido la respuesta individual de los docentes ante la crisis y el cambio en las prioridades, en particular en las escuelas de sectores populares. La importancia no está en los contenidos, en el desarrollo del currículum, sino en la necesidad de mantener activo el vínculo. Notamos que ésta no es una prioridad en las escuelas que atienden a población de clases medias y altas. Como lo señalan algunos de nuestros entrevistados.

Garantizar alimento a las familias y alguna proximidad con la escuela es la tarea....que no se debilite el lazo con los aprendizajes y con los vínculos escolares. (E1)

Nos bajaron medidas de carácter pedagógico: sobre todo mantener el contacto con las familias y revisar las tareas de los chicos, en especial para los que tienen mayores dificultades. Se pide no mandar tareas para toda la semana, sino todos los días, pero sobre todo mantener el contacto con las familias. (E2: vicedirector, escuela gestión pública clase media, CABA)

La inclusión es un tema central de la agenda política educativa, y uno de los principales problemas de exclusión es asegurar la continuidad pedagógica. Por ello, resultan significativos los testimonios de los entrevistados: más que contenidos o desarrollo de ejercicios, es fundamental la contención de la red, la trama que se puede establecer entre las escuelas y la comunidad educativa, evitar el abandono, que se verifica en los estudiantes de los sectores populares. Pero este primer paso, no garantiza la inclusión, y se corre el riesgo de invisibilizar la segregación.

Respecto de la existencia de un plan de contingencia para abordar esta crisis, el Vicedirector una escuela de la zona sur de CABA comentaba:

El único procedimiento de crisis que se hace en las escuelas es de evacuación en caso de desastre, pero no hay un procedimiento bajo las circunstancias que estamos viviendo. (E2)

Esta situación pone en evidencia que las respuestas que se adoptan muchas veces adolecen de anticipación. La acción es lo que prevalece, en los distintos niveles, quizás sin una previsión o instrucciones precisas en base a una planificación u orientación, que tenga en cuenta un horizonte temporal que supere la inmediatez.

Los testimonios dan cuenta de situaciones diferenciales en cuanto al rol que deben asumir los docentes. Algunos han señalado la prioridad en la construcción del vínculo, de la trama que fortalezca la relación entre el establecimiento y la comunidad educativa. En otros casos, la orientación llevó a buscar alternativas de comunicación y producción y distribución de materiales, impresos o en forma virtual.

Desde los niveles centrales, se ponen en marcha un conjunto de acciones, muchas de ellas vinculadas con la producción de recursos pedagógicos diseñados desde una perspectiva propia de sectores medios urbanos con disponibilidad de recursos tecnológicos.

Respecto de la anticipación de la crisis, cuando ya llevaba más de dos meses del brote en China, el Ministerio había comenzado a evaluar la posibilidad de suspensión de las clases. Según sus expresiones: 
Por indicación del presidente, a comienzos de marzo se comenzó a trabajar en una estrategia pedagógica por si había que suspender las clases. Por eso, al momento en que el Presidente anunció la suspensión de clases pudimos presentar el Portal Seguimos Educando, de navegabilidad gratuita, por el compromiso de las empresas de telefonía celular y también lanzar dos programas de televisión en la TV Pública, Educ.ar y Paka Paka de 9 a 11 y de 14 a 16 para educación inicial, primaria y secundario que empezó el mismo día en que se suspendieron las clases. Se registraron 1.400.000 visitas únicas, casi el 70\% por el celular, que habla de un proceso de democratización de acceso. (Ministro de Educación de la Nación)

Como advirtió Paul Virilio (1997), ante la urgencia, la velocidad reemplaza a la comunicación. $\mathrm{Y}$ es en este reemplazo que se pierden de vista las diferencias.

Una docente de escuela de población vulnerable en CABA, comentaba:

En el sistema educativo las cosas, en general, se hacen cuando "bajan" circulares desde
la dirección de primaria/ministerio. Las conducciones como están con mil cosas
accionan cuando vienen los pedidos formales desde arriba. Todo esto fue muy rápido.
Otra cosa, es lo que sucede con los docentes... muchos pudimos armar cuadernillos, otros
entregaron libros con alguna propuesta. Todo esto se resolvió el lunes cuando la
suspensión de clases había sido un hecho. Todo fue muy rápido. Hay docentes que
pudieron armar grupo de wup, otros no. En algunas escuelas hay blogs o se usa la
plataforma edmodo. Muchos pibes no tienen computadoras ni buen acceso a las redes.
Es una situación en la cual estamos atentos, todos, viendo la realidad y las
posibilidades. Es una mirada particular, atenta a las respuestas que van dando las
familias. Yo tengo grupo de whatsapp intento mantener una comunicación casi
cotidiana. Donde busco un ida y vuelta. No es fácil. Es todo ensayo y error. (E1)

Se observa que en la velocidad para reaccionar se pierde de vista la necesidad de una comunicación más profunda que identifique con claridad cuáles son las condiciones reales en que viven amplios sectores de la población cuyos derechos se encuentran vulnerados desde hace muchas décadas. En la distribución de los bienes materiales y culturales muchas veces les ha tocado lo que sobra y se desecha. No todos los estudiantes van a transitar la cuarentena de la misma manera. El riesgo estriba que en la preocupación por la velocidad las decisiones puedan ser vividas como indiferencia por quienes perciben que lo que se produce nuevamente no es para ellos. No es nuevo que la indiferenciación sólo produce desigualdades. Por lo cual es imperativo planificar, diseñar e implementar políticas educativas bajo el principio de igualdad de oportunidades. En este sentido las administraciones deben ser permeables y estar atentas a las necesidades y posibilidades de los y las estudiantes en todos y cada uno de los niveles educativos.

"Poder y velocidad son inseparables al igual que riqueza y velocidad son inseparables. Poder es siempre poder de controlar un territorio con mensajes, modos de transporte y comunicación" (Virilio, 1997, p. 15). El poder de control sobre la acción educativa en la emergencia se encuentra atravesado por la urgencia que se traduce en acciones veloces para dar respuestas inmediatas a necesidades propias de un sistema educativo en funcionamiento, con escuelas que concentran niños pobres y escuelas con niños de clase media y alta.

No se cuestiona la velocidad de las decisiones ni las urgencias para disponer de recursos didácticos para la población escolar. El problema que se advierte, en una primera reacción por sustituir el funcionamiento de la escuela presencial por un funcionamiento virtual, es la invisibilización de las diferentes condiciones de acceso a los recursos digitales. 


\title{
4.3. El curriculum frente a la heterogeneidad de las respuestas frente a la crisis
}

El sistema educativo argentino se caracteriza por su diversidad tanto en tamaño de las instituciones, en su localización, de los recursos que disponen, como por las características socio económicas de la población que atiende. De acuerdo con los testimonios relevados, se hace presente una mayor heterogeneidad a la ya existente.

Algunas escuelas ya trabajaban desde hace tiempo con tecnologías educativas y plataformas de apoyo o complementarias a la presencialidad, no solo en contenidos educativos sino también para la comunicación entre la escuela y la familia. En el otro extremo, otras nunca implementaron o dispusieron de recursos tecnológicos. Ilustra el testimonio de una docente de una de las escuelas que atiende población vulnerable:

\begin{abstract}
To le pregunté a la directora si podía armar un grupo de Whatsapp, porque en mi escuela no hay blog, no hay edmodo, los pibes no tienen computadora, y por mi experiencia que trabajo como capacitador en estos contextos, el vínculo más cercano con las familias es el whatsapp. To le tuve que pedir permiso a la directora si me habilitaba a usar whatsapp, en un primer momento no, después. Esto también genera desigualdades, porque las escuelas de clase media quizás pueden sistematizar más propuestas y las familias pueden estar más atrás, pero también tenemos que ver qué tipo de propuestas mandamos para que puedan hacer con las familias. Hay familias con madres no alfabetizadas, o textos complejos no pueden abordarlos. (E3: maestro de $4^{\circ}$ grado, escuela pública, localización villa de emergencia, clase baja)
\end{abstract}

La heterogeneidad de las respuestas responde a diversos factores: la comunicación u orientación entre docentes y directivos o supervisores, con las familias; la información disponible al momento de la suspensión de clases. Una maestra expresa:

\begin{abstract}
hay mucha heterogeneidad, aún en el interior de la misma escuela. Hay grados en que los maestros no tienen los teléfonos de los alumnos, otros en que sí los tienen y armaron grupos de whatsapp... Por ejemplo, yo soy maestra de segundo grado, y en la escuela hay tres segundos grado. Una no armó el grupo, la otra sí, ayer, y yo la semana pasada... Una maestra armó un cuadernillo. (E4: maestra de segundo segundo grado, escuela pública, localización villa de emergencia zona norte de CABA)

Yo tenía fichas bastante bien armadas de todos, pero nos agarró en los primeros 15 días, muchos no habían comenzado a ir regularmente. Yo tengo un alumno anotado, pero no tengo ninguna información de él porque vino un día, pero está matriculado en la escuela. (E1)
\end{abstract}

Desde el punto de vista de la información, también esta situación de urgencia imposibilitó una implementación ordenada de las distintas estrategias pedagógicas. La disponibilidad de información fue disímil en cada una de las escuelas y los docentes manifestaron las dificultades en el sostenimiento del vínculo con los estudiantes, en particular en aquellas escuelas que no tenían práctica de trabajo on line. Frente a esta situación, en el caso de CABA, el Ministerio de Educación lanzó una campaña de registro de datos de contacto, siendo las familias las que debían inscribirse en la plataforma ${ }^{7}$ indicada. De esta manera se optó por una vía de comunicación centralizada frente a la imposibilidad de las escuelas de establecer el contacto. La precariedad de la situación social profundiza esta brecha, ya que es en la diversidad de las situaciones familiares y condiciones de vida precarias hace que muchas escuelas no hayan podido generar vínculos comunicacionales sólidos, y por ello, la necesidad del llamado público para que los responsables de los estudiantes se pongan en contacto con la institución en que fueran inscriptos. 
A diferencia del caso anterior en que las respuestas son de carácter individual, un directivo de una escuela del Sur de CABA señala:

Cada directivo está coordinando las actividades de ciclo con los maestros y controlando las tareas que se envían por la plataforma edmodo controlar que a todos los chicos les lleguen las tareas. (E2)

Una autoridad escolar que atiende de nivel inicial a nivel secundario manifiesta:

No se recibieron indicaciones para el trabajo durante el aislamiento social por parte de la supervisión. La escuela solo recibió cinco cuadernillos para secundaria y también la indicación para el acceso al sitio del Ministerio de Educación de la Provincia de Buenos Aires.

Por lo tanto, como esto se inició en la segunda semana de clases se decidió para el nivel inicial enviar propuestas de actividades para realizar tres veces por semana, la mayoría orientadas a la prevención de la salud. Al estar en el periodo de adaptación las actividades son recreativas y no curriculares orientadas a transitar el aislamiento. Toda la información para el nivel se canaliza por la página del colegio. La escuela sube las propuestas a la página y las familias acceden allí. Para el nivel primario: dividieron las estrategias por ciclo. Para el primer ciclo se enviaron actividades correspondientes a la etapa diagnóstica de sencilla resolución. Las resoluciones de estas actividades no reciben retroalimentación por parte de los docentes. El representante legal expresa "son actividades sin ida y vuelta". Los alumnos las reciben por whatsapp o por Google Drive. También se generaron correos electrónicos especialmente para mantener la comunicación con los alumnos. Para el segundo ciclo: se incluyeron actividades con retroalimentación por parte del docente. Tipo de actividades problemas matemáticos, lecturas escogidas para trabajar estrategias de comprensión lectora. En el nivel secundario, cada uno de los profesores escoge y diseña actividades. No hay criterios comunes. La comunicación es por correo, Instagram, no hay unificación. No se estableció un criterio común institucional. (E5: directivo escuela privada, localización zona norte del conurbano de la provincia de Buenos Aires, población de clase media)

Esta comunicación da cuenta de una organización institucional que tiene trabajo previo con uso de tecnologías. Como se puede advertir la ausencia de criterios unificados a nivel institucional evidencia una diferenciación por niveles. En otros casos, la diferenciación es de corte individual. Algunos de los testimonios registrados de los entrevistados relatan:

Ya sabíamos el domingo que no iba a haber clases, que se suspendían. El lunes todos tuvimos que ir a la escuela y hubo reunión con el equipo directivo y cada escuela decidió armar lo que quiso, había mucha disparidad... a algunos maestros los mandaban a las casas, otros propusieron turnos de guardias para cubrir hasta el día 31. (E3)

Algunas escuelas tienen blog, entonces dijeron de mandar actividades e información a través de la dirección o al facilitador de intec (facilitador tecnológico), otras escuelas no tienen blog, como la mía. Nadie propuso usar Whatsapp, porque en general se desestimula, y los directores piensan más en lo burocrático, en lo punitivo y evitar conflictos. (E6: vicedirector, escuela privada, todos los niveles educativos obligatorios, zona oeste conurbano bonaerense, población que atiende clase media.)

Lo que llegó a la escuela depende del filtro de cada supervisor, y después de lo que cada director elige, y luego lo que cada maestro puede hacer. (E3)

No tenemos libros, lo que están en la escuela están inventariados y la supervisión de bibliotecas no permiten que salgan de la escuela, porque después no vuelven, y menos en este tipo de escuelas que sale, y después nos quedamos sin libros. (E4)

Como se observa en los testimonios, la disparidad y la heterogeneidad se manifiestan en las características de las escuelas y recursos disponibles en posesión de los docentes y en las formas de hacer frente a la incertidumbre. Sin embargo, hay otra diferenciación 
vinculada con la población que atienden las escuelas. La batería de propuestas que ofrecen las escuelas privadas que atienden sectores medios y altos facilita la continuidad de clases de manera no presencial. Esto se puede observar en el comunicado de una escuela privada que atiende población de clase socioeconómica alta:

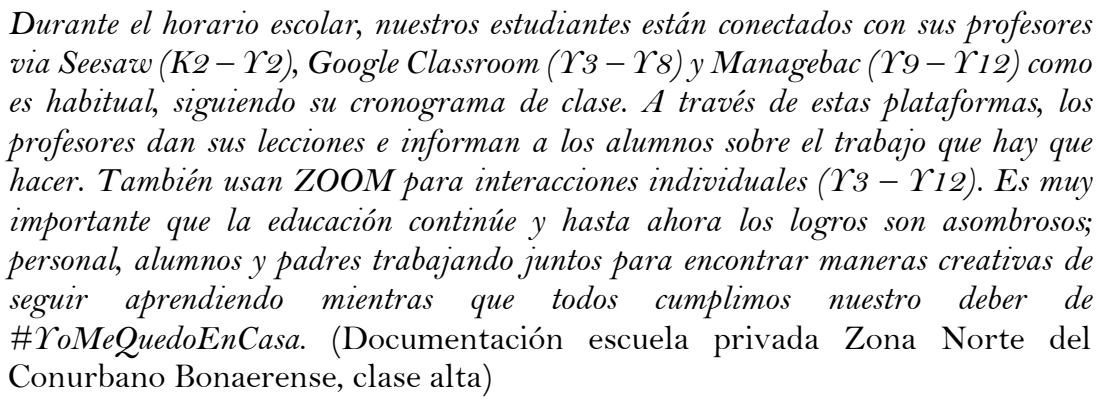

Una directora de escuela primaria privada de comunidad explica:

La escuela atiende a una población que tiene como rasgo común pertenecer a la misma comunidad. Sin embargo, hay familias con altos recursos económicos y otras que reciben ayuda social. Entre estos extremos se ubican familias con diferentes recursos económicos. La escuela tiene una plataforma con la que se trabaja hace varios años, sitios de classroom por sección. Estamos trabajando en jornada extendida con los maestros para que los alumnos dispongan de actividades de aprendizaje mientras dure el aislamiento social. Pero hay casos en que en la familia hay una sola computadora y tienen tres hijos. El padre haciendo teletrabajo y los niños disputándose el turno de uso de la computadora. Los hijos son los últimos en acceder al recurso, ni el telefóno ni las tablets suplen lo que se puede hacer con un ordenador. (E7: directivo escuela privada, población clase media y clase media-alta, CABA)

En esta escuela, de clase media, que atiende a una población algo más heterogénea (no vulnerable) aparece la preocupación por la diferenciación de casos, la necesidad de atender situaciones particulares. Esto requiere un conocimiento de la población atendida, la existencia de un plan institucional. Da cuenta de recursos institucionales que no se observan en otras escuelas.

En las escuelas de sectores populares, el foco es mantener la comunicación con las familias y evitar la desafiliación de los niños con la escuela, en otros casos la resolución de actividades y cómo colaborar con las familias evitando ser punitivos cuando se retrasa o no hay cumplimiento de actividades.

El Ministerio de Educación de la Nación reconoce la problemática de algunas familias (como por ejemplo compartir la computadora padres e hijos) y la situación de los sectores más vulnerables que no tienen posibilidad de conexión, incorporando dispositivos y delineando acciones para garantizar el acceso al conocimiento y el derecho a la educación.

La Argentina es un país que es muy desigual. No es lo mismo un hogar donde hay una maestra hay un profesor, donde hay un hogar donde lamentablemente la familia no tiene capital educativo por más buena voluntad que tenga para acompañar. Ahí tenemos que tener un abordaje, donde la premisa básica es que, en esta situación de excepcionalidad, sabiendo que la escuela es irremplazable, tenemos que lograr que no se profundice la desigualdad. ¿Entonces qué abordaje le queremos dar? Primero la televisión. La televisión ingresa a nuestros hogares, estamos en proceso de preproducción de 7 programas diarios. (...) Porque en una casa puede haber una sola televisión, pero muchos hijos, entonces tenemos que lograr que la oferta llegue en distintos momentos del día para que esas dos horas nos permitan sentarnos frente a la mesa, organizarnos, con los maestros, con el portal. El Portal también va a tener una propuesta curricular diaria. Es un ejercicio que tenemos que hacer todos en familia. 
El Estado tiene que estar presente. El Estado son las provincias, pero también el Estado Nacional. Estamos produciendo cuadernillos para que lleguen a cada uno de los hogares, principalmente los que no tienen conectividad. La radio también es otra herramienta fundamental que nos lo pide la ruralidad de la Argentina. (Entrevista televisiva al Ministro de Educación de la Nación ${ }^{8}$ )

Una cuestión que se vislumbra por la emergencia sanitaria es la disolución del currículum escolar. La edición de cuadernillos en diferentes soportes provoca la separación de los componentes curriculares esenciales y, así, las propuestas escolares diseñadas para el contex to de aislamiento no logran constituir un currículum mínimo obligatorio. Es decir, el conjunto de recursos dispuestos para su distribución a través de diferentes medios e incluso soportes no logran configurar el conjunto de campos disciplinares esenciales para todos los estudiantes.

La situación y resolución de la crisis plantea un dilema: o se define la interrupción de la escolaridad y las propuestas disponibles son recursos a disposición de las familias para que los alumnos los utilicen como "paliativo" o se establece un currículum escolar básico, que defina los aprendizajes indispensables para evitar la discontinuidad de las trayectorias educativas. La vuelta a la escuela debe encontrar a los docentes preparados para considerar mayor heterogeneidad y contar con orientaciones claras para enfrentar las desigualdades.

Un desarrollo curricular que se despliega en contexto de aislamiento, sin el suficiente planeamiento del nivel central, sin la suficiente preparación de las escuelas, tanto para los docentes, como para los estudiantes, no solo es una limitante por el acceso a los recursos sino también por la restricción que impone a las interacciones propias de las prácticas de aula. Los especialistas en educación virtual sostienen la riqueza que pueden albergar estas experiencias, pero requieren criterios de desarrollo particular. No se trata de volcar en una página web el ejercicio de la clase presencial.

El diseño de actividades de producción centralizada, más allá de la disponibilidad de los recursos, acota el territorio curricular ya que la "velocidad" impuesta para disponer de dichos recursos, la distancia, la virtualidad y el aislamiento restringe el papel creativo de los docentes en relación con el tiempo para la planificación y con las interacciones propias de la práctica de enseñanza en un contexto escolar particular.

¿Es posible pensar en un currículum sin contexto escolar para la educación obligatoria? El papel regulador del currículum se verá debilitado ante la ausencia de la experiencia colectiva en la producción de aprendizajes. Se restringen aún más las condiciones de accesibilidad a fuentes de información necesarias para evitar la simple realización de “ejercitaciones” concibiendo que su recurrencia es garantía de aprendizajes.

Al mismo tiempo disminuye el poder del profesorado a quienes se los excluye de la acción de programación y con ello se ven menguadas sus posibilidades de anticipación. No es posible prever qué sucederá durante la implementación de las actividades propuestas centralmente y cuál será efectivamente el rol que podrán desempeñar.

No se trata de una oposición a las decisiones de las administraciones de proveer recursos didácticos para el aprendizaje, ni de la importancia de dar continuidad al vínculo entre la institución educativa, los estudiantes y la familia. Advertimos los riesgos de concebir que con solo acceder a dichos recursos se garantiza una educación justa. Un aspecto

${ }^{8}$ Entrevista realizada el 25 de marzo de $2020, \mathrm{C} 5 \mathrm{~N}$. 
importante de esta diferencia es que quienes menos tienen quedarán más alejados no sólo de aquellos recursos materiales que posibilitan el acceso sino también de los simbólicos pues se producen mayores limitaciones de ambas fronteras: simbólicas y materiales.

Hola profe, mire, a mí el celular me anda para el culo, que le voy a decir... yo no puedo ver las imágenes, no puedo ver nada... y no me puede obligar a mí a que el Lucas copie todas las cosas porque no tengo un buen teléfono. Encima tenemos un solo teléfono. No me pueden obligar que el Lucas haga las tareas... Si fuese por mí, que vaya a la escuela, que es lo que yo quiero, que estén en la escuela y no en la casa.... si por algo le compré todo el material... después que se fije él, el Lucas, cómo puede copiar las tareas, porque lo que dijo el presidente que nadie puede salir de la casa, estamos todos en cuarentena y nos quieren mandar a un cyber a sacar las tareas. $Y_{0}$ les digo, las faltas mejor que no se las pongan al Lucas por no hacer las tareas. (Mensaje de una madre al maestro de su hijo, E3)

Este testimonio da cuenta de los desafíos que deben asumir las escuelas.

Esta situación también genera desigualdades, porque las escuelas de clase media quizás pueden sistematizar más propuestas y las familias pueden estar más atrás, pero también tenemos que ver qué tipo de propuestas mandamos para que puedan hacer con las familias. Hay familias con madres analfabetas, los textos complejos no pueden abordarlos yo tengo segundo grado, ¿pero los alumnos de séptimo? ¿Cómo hacemos?

(E4)

Una coincidencia en los documentos y testimonios es que el centro en el cual se toman las decisiones de política educativa se encuentra en el Estado. Estas preguntas no se cierran en el período del aislamiento. ¿Qué pasará con lo que se intentó enseñar y aprender cuando se vuelva a la escuela? ¿De qué forma se retomará? ¿Cómo se articulará con lo que resta del año? Será importante, promover investigaciones que sigan estrechamente el análisis de la segregación educativa considerando la creciente virtualización de la educación como así también las consecuencias de la ruptura del proceso pedagógico y acceso al conocimiento. Asimismo, será relevante disponer de estudios que aporten evidencias cuantitativas y cualitativas a la desigualdad de acceso a las tecnologías.

\section{Discusión}

Es necesario señalar la profundidad y extensión de la crisis en curso. Esta situación evidencia que en los distintos sectores hay posibilidades diferentes de concretar las instrucciones de las autoridades que ponen de manifiesto las diferentes capacidades de los establecimientos educativos para dar continuidad pedagógica. En este marco, los sectores vulnerables se vuelven aún más vulnerables.

En términos de planeamiento, si bien desde el inicio de la pandemia transcurrieron cerca de cuatro meses hasta la suspensión de las clases, las acciones hasta dicha decisión fueron reactivas y descoordinadas. Si antes de la situación de crisis el diagnóstico del sistema educativo estaba marcado por la segregación, este tipo de eventos potencia las consecuencias de los déficits existentes y da cuenta de cómo las desigualdades se agravan. Aunque hay en el discurso oficial una intención de continuidad de las clases para toda la población, los testimonios dan cuenta de las diferencias en infraestructuras, capacidades técnicas y estrategias pedagógicas.

Si bien los modelos renovados de planeamiento contemplan la flexibilidad y el ajuste a las situaciones, este nuevo escenario parece transportarnos a una situación diferente: la del manejo de crisis, con lógicas propias como la de reducción de los daños. 
Las discusiones abiertas sobre el calendario escolar futuro, el cumplimiento de los 180 días de clases regulados por ley, las evaluaciones y acreditaciones y la continuidad con el ciclo lectivo del año siguiente parece ser el comienzo de un nuevo ciclo de planeamiento destinado a recomponer las consecuencias de la pandemia.

No se puede soslayar que la centralidad en las decisiones, en un caso así, corresponderá al ámbito de la salud. Sin embargo, el sector educación, aun cuando su recuperación en términos de "vuelta a la normalidad" sea más lento, no puede dejar de ser considerado esencial ni estratégico incluso para el resto de la sociedad, aunque los resultados no sean inmediatos. En términos de segregación, la autonomía de las instituciones y la ausencia de estrategias coordinadas profundizan estas diferencias. La promesa de la virtud de la autonomía escolar para atender las necesidades locales se desvanece frente a la disparidad de recursos y apoyos para responder las necesidades de diferentes grupos sociales. Los más vulnerables vuelven a ver limitados sus derechos y sus posibilidades de hacer efectiva la igualdad y equidad educativas tan pregonadas en los discursos neoliberales.

Las respuestas institucionales diferenciales muestran una relación significativa con la situación económica objetiva de las poblaciones a las que atiende cada escuela, y lo público y lo privado vuelven a jugar un rol importante. Se observó que las escuelas del sector privado de élite pudieron articular programas de continuidad que suponen, además de los recursos por parte de la escuela, un aporte importante del lado de las familias en contraposición con algunas escuelas del sector público que no disponían ni de recursos ni posibilidad de acompañamiento familiar.

El supuesto de la tecnología como superador de las dificultades, puede ser considerado para ciertos sectores y con determinadas condiciones. Pero no se cumple esta condición en la totalidad de las escuelas y, particularmente, en las de los sectores vulnerables, incidiendo en la profundización de la diferenciación de los segmentos. Pretender la normalidad en tiempo de excepción es una utopía. La escolaridad aporta normalidad a la vida de niños y adolescentes. El pasaje de dispositivos presenciales a virtuales no es una garantía per se de la educación.

Demasiadas variables atentan contra la continuidad de la normalidad: el tiempo escolar, las exigencias o condiciones laborales de docentes y padres, el tiempo doméstico y el tiempo recreativo. Todas ellas en situaciones críticas se superponen.

Finalmente, la reflexión acerca del curso de las acciones en el ámbito educativo en este breve período pone de manifiesto la segregación escolar en Argentina (preexistente a la situación de excepcionalidad). Esta evidencia podría ser utilizada por las administraciones educativas para la toma de decisiones a futuro orientadas a la formulación de políticas para la generación de una educación más inclusiva, justa e igualitaria. En ese sentido, poner en primer plano las condiciones de partida respecto de las capacidades que tienen las escuelas para la innovación es un aspecto central que no puede ser invisibilizado. Para comprender y evaluar las acciones es necesario volver a poner el eje en la función de la escuela: la distribución social del conocimiento. Paradójicamente, mientras los sistemas educativos creados en siglo XIX se preocupan por su expansión y cobertura, sin necesidad de explicitar cómo lograrlas, hoy aparece la distribución del saber como un mecanismo restringido, donde prevalece la responsabilidad de la familia en disponer o no de computadora u otro dispositivo para tener acceso a bienes simbólicos y culturales. 


\section{Referencias}

Angulo, J. F. (1994). El gato por la liebre o la descentralización en el sistema educativo español. Cuadernos de Pedagogía, 222, 74-83.

Bonfiglio, J., Veram J. y Salvia, A. (2019). Pobreza monetaria y vulnerabilidad de derechos. Inequidades de las condiciones materiales de vida en los hogares de la Argentina urbana (2010-2018). Educa.

Braslavsky, C. (1985). La discriminación educativa en Argentina. FLACSO.

Brito, A. (2015). Nuevas coordenadas para la alfabetización: Debates, tensiones y desafíos en el escenario de la cultura digital. IIPE- UNESCO.

Cobo, C. (2016). La innovación pendiente. Reflexiones (y provocaciones) sobre educación, tecnología y conocimiento. Debate.

Da Silva, T. T. (1988). Cultura y currículum como prácticas de significación. Revista de Estudios del Currículum, 1(1), art 6.

Gasparini, L., Jaume, D., Serio, M. y Vázquez E. (2011). La segregación entre escuelas públicas y privadas en Argentina. Reconstruyendo la evidencia. Revista Desarrollo Económico, 51(202), 35-57.

Kessler, G. (2002). La experiencia escolar fragmentada. Estudiantes y docentes en la escuela media en Buenos Aires. IIPE-UNESCO.

Kessler, G. (2014). Controversias sobre la desigualdad. Argentina, 2003-2013. FCE.

Kliksberg, B. (2005). América Latina: La región más desigual de todas. Revista de Ciencias Sociales, $11(3), 411-421$.

Krüger, N. (2013). Segregación social y desigualdad de logros educativos en Argentina. Archivos Analíticos de Políticas Educativas, 21, 1-26. https://doi.org/10.14507/epaa.v21n86.2013

Krüger, N. (2014). Más allá del acceso: segregación social argentino. Cuadernos de Economía, 33(63), 513-542. https://doi.org/10.15446/cuad.econ.v33n63.45344

Krüger, N. (2019). La segregación por nivel socioeconómico como dimensión de la exclusión educativa: 15 años de evolución en América Latina. Archivos Analíticos de Políticas Educativas, 27(8), 35-67. https://doi.org/10.14507/epaa.27.3577

Lugo, M. T. (2016). Entornos digitales y políticas educativas: Dilemas y certezas. IIPE- UNESCO.

Maggio, M. (2012) Entre la inclusión digital y la recreación de la enseñanza: el modelo 1 a 1 en Argentina. Revista Iberoamericana de Educación, 79(1), 11-31.

Maggio, M. (2019) La reinvención colectiva de las prácticas de la enseñanza como desafío de la educación latinoamericana contemporánea. Cuadernos de Pedagogía, 500, 146-150

Marchesi, A. (2000). Un sistema de indicadores de desigualdad educativa. Revista Iberoamericana de Educación, 23, 67-88.

Marés Serra, L. (2012). Panorama regional de estrategias uno a uno: América Latina + el caso de Argentina. Educ.ar.

Meirieu, P. (2020). La escuela después... ¿̨con la pedagogía de antes? Movimiento cooperativo de escuela popular. http://www.mcep.es/2020/04/18/la-escuela-despues-con-la-pedagogia-de-antesphilippe-meirieu/

Murillo, F. J. (2016) Midiendo la segregación escolar en América Latina. Un análisis metodológico utilizando el TERCE. REICE. Revista Iberoamericana sobre Calidad, Eficacia y Cambio en Educación, 14(4), 33-60. https://doi.org/10.15366/reice2016.14.4.002 
Murillo, F. J. y Martínez-Garrido, C. (2017). Estimación de la magnitud de la segregación escolar en América Latina. MAGIS. Revista Internacional de Investigación en Educación, 9(19), 11-30. https://doi.org/10.11144/Javeriana.m9-19.emse

Neiman, G. y Quaranta, G. (2006). Los estudios de caso en la investigación sociológica. Estrategias de Investigación Cualitativa, 1, 213-237.

Piscitelli, A. (2009). Nativos digitales. Dieta cognitiva, inteligencia colectiva y arquitecturas de participación. Santillana.

Sen, A. y Kliksberg, B. (2007). Primero la gente: Una mirada desde la ética del desarrollo a los principales problemas del mundo globalizado. Ediciones Deusto.

Sunkel, G., Trucco, D. y Espejo, A. (2014). La integración de las tecnologías digitales en las escuelas de América Latina y el Caribe. Una mirada multidimensional. CEPAL.

Tiramonti, G. (2004) La trama de la desigualdad educativa. Mutaciones recientes en la escuela media. Manantial.

Valenzuela, J. P., Bellei, C. y Ríos, D. D. L. (2014). Socioeconomic school segregation in a marketoriented educational system. The case of Chile. Journal of Education Policy, 29(2), 217-241. https://doi.org/10.1080/02680939.2013.806995

Virilio, P. (1997). El cibermundo. La política de lo peor. Cátedra.

\section{Breve CV de los autores}

\section{Marisa Álvarez}

Licenciada en Administración (UBA), Magíster en Dirección de Sistemas de Información (USAL-SUNNY at Albany), Doctoranda en Educación (UNTREF/UNLA/UNSAM). Profesora titular en la Maestría de Política y Administración de la Educación de UNTREF. Investigadora del Núcleo Interdisciplinario de Formación y Estudios para el Desarrollo de la Educación (NIFEDE) en la UNTREF. Profesora adjunta del Departamento de Ciencias de la Educación de la Facultad de Filosofía y Letras de la UBA. Cátedras: Administración de la Educación y Planeamiento Educacional. Editora de la Revista Argentina de Educación Superior. Especialista en administración y planeamiento de la educación, se ha especializado en gestión y evaluación de programas educativos. ORCID ID: https://orcid.org/0000-0001-5804-1962. Email: malvarez@untref.edu.ar

\section{Natalia Gardyn}

Licenciada en Ciencias de la Educación (UBA). Maestranda en Administración Pública (UBA). Docente de las Cátedras de Administración de la Educación y Planeamiento Educacional del Departamento de Ciencias de la Educación e Investigadora del Instituto de Investigaciones de Ciencias de la Educación de la Facultad de Filosofía y Letras de la Universidad de Buenos Aires. Coordinadora académica de la Maestría en Docencia Universitaria de la Facultad Regional General Pacheco de la Universidad Tecnológica Nacional. Asesora Pedagógica en universidades públicas y privadas. Se ha especializado académicamente en temas de administración de la educación, financiamiento educativo y planeamiento, gestión y evaluación universitaria. ORCID ID: https://orcid.org/oooo0002-8579-2929. Email: natalia.gardyn@gmail.com 


\begin{abstract}
Alberto Iardelevsky
Licenciado y Profesor en Ciencias de la Educación. Universidad de Buenos Aires, Doctorando en Educación (UNTREF/UNLA/UNSAM). Profesor titular en la Maestría de Política y Administración de la Educación de UNTREF. Profesor Adjunto regular del Departamento de Ciencias de la Educación del Departamento de Ciencias de la Educación. Facultad de Filosofía y Letras. Cátedra: Administración de la Educación. Universidad de Buenos Aires. Universidad Nacional de Chilecito. Investigador. Profesor Titular de Didáctica II Universidad Nacional de Lomas de Zamora. Se ha especializado académicamente en temas de currículum, formación de docentes y en formación en seguridad. ORCID ID: https://orcid.org/0000-0003-2313-4768. Email: iardeal@hotmail.com
\end{abstract}

\title{
Gabriel Rebello
}

Licenciado en Ciencias de la Educación (UBA). Doctorando en Educación (UNTREF/UNLA/UNSAM). Profesor Adjunto de Planeamiento y Gestión de Universidades (UBA). Profesor Titular de Planificación Educativa (UNSI). Investigador Instituto de Investigaciones en Ciencias de la Educación (UBA). Se ha especializado en investigación de política y gestión de la educación. ORCID ID: https://orcid.org/ooooo001-7270-139X. Email: grebello@gmail.com 